\title{
Frequency Analysis of the Detectability of Virtual Haptic Gratings
}

\author{
Steven Cholewiak and Hong Z. Tan \\ Haptic Interface Research Laboratory \\ Purdue University, West Lafayette, IN, USA \\ E-mail: \{sac; hongtan\}@purdue.edu
}

\begin{abstract}
The tactile detectability of sinusoidal and squarewave virtual texture gratings were measured and analyzed. Using a three-interval one-up three-down adaptive tracking procedure, detection thresholds for virtual gratings were estimated using a customdesigned high position-resolution 3-degrees-offreedom force-feedback haptic device. Two types of gratings were used, defined by sinusoidal and square waveforms, with spatial wavelengths of 0.2 to 25.6 $\mathrm{mm}$. The results indicated that the participants demonstrated a higher sensitivity (i.e., lower detection threshold) to square-wave gratings than to sinusoidal ones at all the wavelengths tested. When the squarewave gratings were represented by the explicative Fourier series, it became apparent that the detectability of the square-wave gratings could be determined by that of the sinusoidal gratings at the corresponding fundamental frequencies. This was true for any square-wave grating as long as the detection threshold for the fundamental component was below those of the harmonic components.
\end{abstract}

\section{Introduction}

Many studies of human texture perception, including our own, have employed sinusoidal texture models for the reason that any surface height functions representing texture can be decomposed into a series of sinusoidal functions using Fourier analysis [1-4]. The implicit assumptions were that (1) the human somatosensory system may be capable of performing a spectrum analysis of the proximal stimuli arising from interacting with a textured surface, and (2) the perception of individual spectral components may be linearly combined to form an overall percept. That these assumptions may have some validity was first suggested by a study on visual grating perception [5] Using gratings of sine, square, rectangular and saw- tooth waveforms displayed on a TV monitor, the authors provided evidence for a linear system model of visual perception that operates within a limited range of frequencies.

One type of tactile analog of the visual gratings used in the Campbell and Robson study [5] is surfaceheight gratings at the microscale (i.e., surface texture). In the present study, we used virtual surfaces with sinusoidal and square-wave gratings to analyze the detectability of the gratings in the frequency domain. Specifically, we measured the detection thresholds for sinusoidal and square-wave gratings at a series of spatial wavelengths / frequencies. We then compared the measured detection thresholds for square-wave gratings to those predicted from the detectability of the corresponding fundamental and harmonic components using thresholds measured for sinusoidal gratings.

We outline the main idea of the present study as follows. Let us consider two gratings, one sinusoidal and one square-wave, with the same amplitude (see Fig. 1). The height map of the sinusoidal gratings is defined by

$h_{\sin }(x)=A \sin (2 \pi x / \lambda)$,

Eq. 1

while the height map of the square-wave gratings is defined by

$h_{\text {square }}(x)=\left\{\begin{array}{cl}A, & \text { if } \sin (2 \pi x / \lambda)>0 \\ -A, & \text { if } \sin (2 \pi x / \lambda) \leq 0\end{array}\right.$,

Eq. 2

where $A$ denotes the amplitude of the waveforms, $x$ denotes the $x$-position along which surface height varies, and $\lambda$ denotes the spatial wavelength of the virtual gratings. The terms texture and grating are used interchangeably in this article to describe the virtual surfaces produced by the calculated height maps.

The Fourier expansion of the square-wave texture shown in Eq. 2 can be expressed as

$$
A\left(\frac{4}{\pi}\right)\left\{\sin \frac{2 \pi x}{\lambda}+\frac{1}{3} \sin 3 \frac{2 \pi x}{\lambda}+\frac{1}{5} \sin 5 \frac{2 \pi x}{\lambda}+\cdots\right\} \text {, Eq. } 3
$$




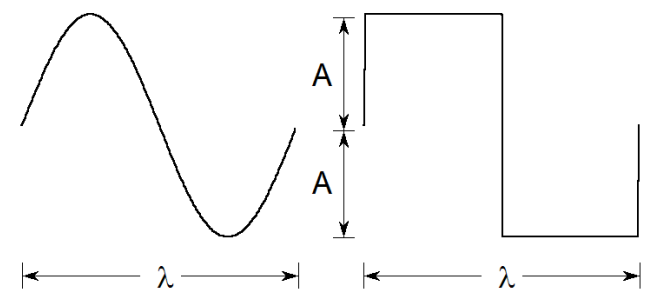

Figure 1. The sinusoidal and square-wave gratings used in the present study. Shown are one cycle of each waveform, respectively, where $A$ denotes the amplitude and $\lambda$ the wavelength of the waveforms.

It now follows that the intensity of the fundamental component in a square-wave grating is slightly larger than that of the sinusoidal grating by a factor of $4 / \pi$, provided that the sinusoidal and square-wave gratings have the same amplitude, as is the case in Fig. 1. If we assume that the spectral components shown in Eq. 3 can be sensed individually by a linear somatosensory system, then the detectability of a square-wave grating should be determined by the most detectable component. Furthermore, the detectability of the components can be determined by the detection thresholds for sinusoidal gratings at the corresponding spatial wavelength/frequency, after taking into account the coefficients in Eq. 3 (e.g., $4 / \pi$ for the fundamental component, $4 /(3 \pi)$ for the third harmonic, etc.). It should now be clear that once the detection thresholds for sinusoidal gratings have been measured, the relative detectability of the spectral components in a square-wave grating can be calculated according to Eq. 3. The prediction can then be compared to the experimentally-measured detection threshold for square-wave gratings. For example, suppose we have determined that the fundamental component of a square-wave at wavelength $\lambda$ has the lowest detection threshold, we can then predict that the detection threshold in amplitude for a square-wave grating at wavelength $\lambda$ should be $\pi / 4$ times the detection threshold of a sinusoidal grating at wavelength $\lambda$.

Two experiments were conducted in the present study. The first experiment measured the detection thresholds for sinusoidal gratings. The second experiment measured the same for square-wave gratings at the same selected wavelengths. In Sections 2 and 3, we present the experimental methods and results, respectively. Section 4 concludes the article with a brief discussion.

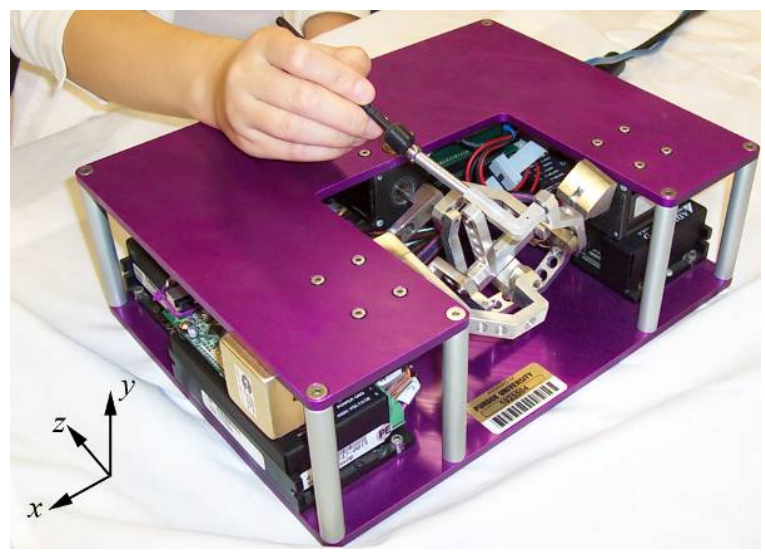

Figure 2. The mini-stick, a 3-DOF forcefeedback device used to render virtual gratings in the present study.

\section{Methods}

\subsection{Participants}

Three participants took part in the present study (two males and one female, age range 22-41 years old, average age 29 years old). All were right-handed by self report and had previous experience with haptic interfaces and experiments. The participants gave their written consent to the experimental protocol that had been approved by the Institutional Review Board at Purdue University.

\subsection{Apparatus}

A custom-designed, high position-resolution, 3-DOF force-feedback device (the "mini-stick", see Fig. 2) was used in all the experiments [6]. The mini-stick has a typical position resolution of $1 \mu \mathrm{m}$. Its force commands are updated at $2 \mathrm{kHz}$. A user interacts with the virtual objects rendered by the mini-stick using a stylus. The stylus tip is modeled as a point (i.e. infinitesimally small). Results from an earlier study have shown that the discrimination thresholds using real texture samples and virtual textures simulated on the mini-stick were very similar, thereby indicating that the mini-stick haptic interface device appears to be an appropriate device for studying haptic texture perception [7]. 


\subsection{Stimuli}

Virtual textures with sinusoidal and square-wave gratings were generated on the mini-stick. The surface height for these gratings varied along the $x$-axis of the mini-stick coordinate frame but remained constant along the $z$-axis (see Fig. 2 for an illustration of the mini-stick and definition of the coordinates). The height map of the sinusoidal and square-wave gratings were defined by Eq. 1 and Eq. 2 shown earlier. The restoring force was then calculated for both types of textures as follows:

$F_{y}=\left\{\begin{array}{cl}K \times[h(x)-y], & \text { if } y<h(x) \\ 0, & \text { if } y \geq h(x)\end{array}\right.$

where $y$ denotes the vertical position ( $y$-position) of the mini-stick stylus tip (see Fig. 2), and $K$ denotes the stiffness constant ( $K=2.0 \mathrm{~N} / \mathrm{mm}$ in all conditions). It follows that the restoring force was always along the positive direction of the $y$-axis of the mini-stick coordinate system. No forces along the $x$ or $z$ directions were rendered (i.e., no friction forces were generated). It is generally undesirable to render "sharp" force changes by a force-feedback device as was required to generate the square-wave gratings used in the present study. However, we observed no "buzzing" noise or device instability presumably because the gratings were presented at levels close to detection thresholds $(<10 \mu \mathrm{m}$ for square-wave gratings).

For both sinusoidal and square-wave gratings, eight stimulus wavelengths were used: $25.6,12.8,6.4,3.2$, $1.6,0.8,0.4$, and $0.2 \mathrm{~mm}$. These eight spatial wavelengths corresponded to spatial frequencies, described as cycles per millimeter $(\mathrm{c} / \mathrm{mm})$, of $0.0390625,0.078125,0.15625,0.3125,0.625,1.25$, 2.5 , and $5 \mathrm{c} / \mathrm{mm}$. This range was selected to include wavelengths that would provide a wide variety of spatial and temporal frequencies within the constraints of the resolution and workspace of the mini-stick.

\subsection{Procedures}

Both experiments employed a three-interval, forced-choice, one-up three-down adaptive procedure [8-9]. On each trial, the participants received three presentations of stimuli (three intervals). A nontextured flat surface (i.e., $A=0$ ) was presented during two of the three intervals, and a textured surface was presented in the remaining interval. The interval that contained the textured stimulus was randomly chosen to be the first, second, or the third interval. Participants were told to report the interval (" 1 ", " 2 " or "3") in which they felt a texture. Depending on the experimental condition, either a sinusoidal surface or a square-wave surface was presented.

The amplitude of the textured surface was adjusted according to the accuracy of the participant's responses. On the first trial of each session, the initial amplitude was chosen to be approximately $20 \mathrm{~dB}$ above the expected threshold level to insure that the participants clearly felt the stimulus. The amplitude level was decreased after three consecutive correct responses, and was increased after one incorrect response. Initially, the amplitude level changed by 6dB. The relatively large step size allowed the participants to approach their thresholds quickly, facilitating convergence. A reversal occurred when the amplitude level changed from increasing to decreasing (or vice versa). After the first three reversals, the amplitude level changed by $1 \mathrm{~dB}$ for the remaining twelve reversals. The smaller step size improved the precision of the threshold estimates. The experimental session terminated after 12 reversals at the $1 \mathrm{~dB}$ step size.

The number of trials in each session varied as the participant's performance converged on the threshold for the specific spatial frequency. Each session typically took 60 to 80 trials, lasting 10 to 15 minutes. The participant was asked to repeat a session if the data failed to converge as judged by the experimenter. The total experimental time for each participant to complete all frequencies averaged 4 to 5 hours. This design ensured that the data reflected the asymptotic threshold performance of a few representative experienced participants and were relatively free of learning effects.

There were two experiments: detection of virtual textures modeled with sinusoidal gratings (Exp. 1) and those with square-wave gratings (Exp. 2): Exp. 1 was conducted before Exp. 2 for all participants. During each experimental session, participants were tested on one of the stimulus wavelengths selected in randomized order. Each participant provided one threshold measurement for each combination of waveform and wavelength $(2$ waveforms $\times 8$ wavelengths per waveform).

Participants were comfortably seated before a computer screen and keyboard with the dominant arm rested on a comfortable support. The probe was grasped by the dominant hand in the same way that the participant normally held a pencil, and a series of representative stimuli were presented to familiarize the participant with the virtual textured surfaces. The participants were instructed to explore within the center of the mini-stick workspace to avoid non-linear effects present at the boundaries of the workspace. They were encouraged to experiment with, and then 
use the optimal stroking speed that seemed to facilitate their detection of virtual gratings.

The first interval in a trial was initiated by a keystroke on the computer keyboard. The participants were then allowed to move the stylus freely, from side to side, to stroke the virtual surface. When the participant was ready to move on, additional keystrokes initiated the second, then the third intervals. The response indicating the interval that contained the textured surface was recorded by a final keystroke on the number 1, 2, or 3 keys, which initiated the next trial. As is typical with threshold studies, no feedback was provided. The participant was allowed to take a brief break between experimental sessions.

\subsection{Data Analysis}

For each stimulus frequency, detection thresholds were calculated from the peak and valley amplitude values over the last twelve reversals at the $1 \mathrm{~dB}$ step size. Specifically, six threshold values were estimated by averaging the six pairs of peak/valley $A$ values used during the last 12 reversals. The mean and the standard error for the detection threshold were then calculated from the six threshold estimates. According to Levitt [8], the results converged on the psychometric function at the 79.4\%-percentile level.

The detection thresholds measured for a sinusoidal grating (Exp. 1) and a square-wave grating (Exp. 2) at the same wavelength were then used to produce an estimate of their ratio. Each ratio was compared to the coefficients in Eq. 3 to gain insight into which spectral component of a square-wave grating was responsible for the detection of the square-wave grating.

\section{Results}

The detection thresholds averaged over the three participants are shown, as a function of spatial frequency, in Fig. 3. It is apparent that the thresholds for the square-wave virtual gratings (filled squares, Exp. 2) were lower than those for the sinusoidal gratings (open circles, Exp. 1) at all eight spatial frequencies corresponding to the eight tested spatial wavelengths. For both types of gratings, thresholds generally decreased as spatial frequency increased, reached a minimum at around $1.25 \mathrm{c} / \mathrm{mm}$ (wavelength $0.8 \mathrm{~mm}$ ), and increased again at higher spatial frequencies. The thresholds values ranged 1.8-107.4 $\mu \mathrm{m}$ for the sinusoidal gratings and $1.3-6.8 \mu \mathrm{m}$ for the square-wave gratings, with the lowest thresholds approaching the resolution constraints of the mini-stick $(1 \mu \mathrm{m})$. The relatively large standard errors for the

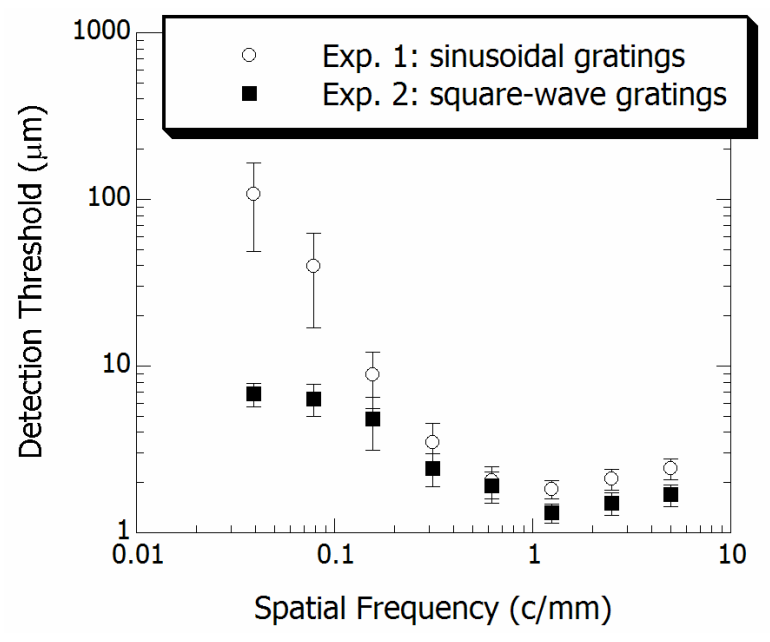

Figure 3. Detection thresholds from Exp. 1 (open circles for sinusoidal gratings) and Exp. 2 (filled squares for square-wave gratings) at eight spatial frequencies. Shown are the averages and standard errors of the thresholds for the three participants.

sinusoidal gratings at the two lowest spatial frequencies were due to the much higher thresholds obtained with S2 at these two largest wavelengths. The difference between the thresholds for the sinusoidal and square-wave gratings of the same wavelength decreased asymptotically as spatial frequency increased (see Fig. 3).

A two-way ANOVA with type of waveform (sinusoidal vs. square-wave) and spatial frequency ( 8 values) as factors was calculated. Each waveform and wavelength pair contained eighteen threshold estimates ( 6 threshold estimates $\times 3$ participants). The ANOVA confirmed that the detection thresholds for the squarewave gratings $(M=3.36, S D=2.64)$ were significantly lower than those for the sinusoidal gratings $(M=$ $20.99, S D=51.50)[F(1,272)=29.73, p<0.001]$. The ANOVA also confirmed a highly significant interaction between the two factors $[F(7,272)=14.97$, $p<0.001]$. Further t-tests confirmed that all of the thresholds for the square-wave gratings were significantly lower than those for the sinusoidal gratings, except at $0.625 \mathrm{c} / \mathrm{mm}$ (wavelength $1.6 \mathrm{~mm}$ ).

The ratios of the thresholds, calculated as the threshold for the sinusoidal grating over the threshold for the square-wave grating at the same spatial frequency, are shown in Fig. 4. The dashed line corresponds to $4 / \pi \approx 1.273$; i.e., the predicted ratio assuming that the detectability of a square-wave grating was determined by the detectability of its 


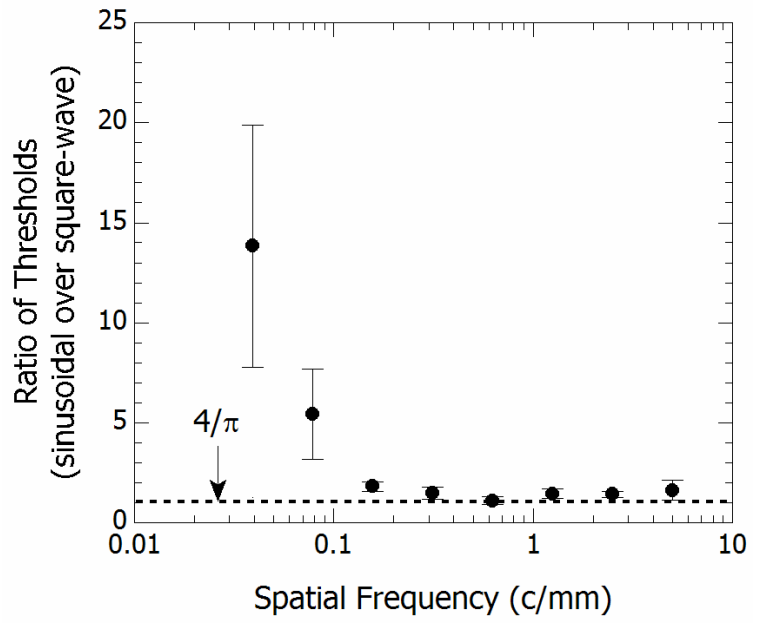

Figure 4. The ratio of detection thresholds (filled circles) for sinusoidal gratings over thresholds for square-wave gratings at the eight spatial frequencies tested. Shown are the averages and standard errors of the ratios from the three participants.

fundamental component. A visual inspection suggests that the ratios did not systematically deviate from the predicted value except at the two lowest spatial frequencies. Z-tests verified that the ratios corresponding to the lowest four spatial frequencies $(0.0390625-0.3125 \mathrm{c} / \mathrm{mm})$ were significantly different from the predicted ratio of $4 / \pi(p<0.001)$, while the ratios corresponding to the highest four spatial frequencies $(0.625-5 \mathrm{c} / \mathrm{mm})$ were not significantly different from $4 / \pi(p>0.05)$. The relatively large standard errors at the two lowest frequencies were again due to inter-subject differences.

\section{Discussion}

The present study measured the detection thresholds for virtual sinusoidal and square-wave texture gratings generated by a 3-DOF, high position-precision forcefeedback device. The results are analyzed in the frequency domain using Fourier series expansion of square-wave gratings. There was evidence that, for most of the wavelengths tested, the detection of square-wave gratings could be predicted by the detectability of the corresponding fundamental frequencies. In other words, the detection threshold for a square-wave grating is related to the threshold for a sinusoidal grating at the corresponding fundamental frequency by the ratio $4 / \pi$, the coefficient for the fundamental component of the square-wave grating.
At the lowest spatial frequencies (or the longest wavelengths), the threshold ratio deviated significantly from $4 / \pi$, indicating that the detection of square-wave may be determined by the detectability of its harmonics, rather than the fundamental component. This is due to the fact that at a low spatial frequency, $\omega$, the detection threshold for a sinusoidal grating at three or five times $\omega$ may be lower than the threshold at $\omega$, even after the coefficients of $1 / 3$ and $1 / 5$ have been applied to the third and fifth harmonics (see Eq. 3 ). Due to the relatively large standard errors observed for the threshold ratios at the lower spatial frequencies (Fig. 4), we were unable to determine quantitatively whether the ratios corresponded to the detection of the harmonics of the square-wave gratings.

There is a large body of literature on the properties of mechanoreceptors in the skin, covering a range of topics from detection thresholds [10-11] to functional mechanisms [12]. Many have investigated the perception of gratings [13-15]. To the best of our knowledge, however, our present study is the first to explicitly investigate the linearity of the somatosensory system using Fourier analysis. The results of our study have implications for not only the mechanism of haptic perception but engineering applications. If the human somatosensory system performs a spectrum analysis of proximal stimuli, as our data suggest, then thresholds for complex signals could potentially be predicted from observed harmonic amplitudes and known sinusoidal waveform thresholds. From an application point of view, it may not be necessary to transmit the higher-frequency components of a haptic signal if the amplitudes of the components are below human detection thresholds. This perception-based approach can greatly ease the requirements on data transmission bandwidth without sacrificing the perceived quality of haptic signals at receiver's end.

Future work will aim to reduce the large standard errors associated with detection thresholds measured at low spatial frequencies (Fig. 3) by using more participants of the same age group. To investigate the possibility of an ordering effect on the data, i.e., the thresholds for sinusoidal gratings were higher than those for square gratings because the latter were measured after the former, the two experiments will be repeated where the waveform as well as the wavelength will be randomized per participant. Additional threshold sessions will be collected to identify and quantify learning effects, if present. Furthermore, we will investigate the perception of more than one spectral component by measuring the threshold at which square-wave gratings no longer feel like sinusoidal gratings. 


\section{Acknowledgment}

This work was supported partly by the National Science Foundation under grant nos. 0328984 and 0533908 , and partly by a NASA award under grant no. NCC 2-1363. The authors wish to thank Dr. Roger Cholewiak for many useful discussions on the present study, and for his careful readings of the manuscript.

\section{References}

[1] S. Choi and H. Z. Tan, "Perceived instability of virtual haptic texture. I. Experimental studies," Presence: Teleoperators and Virtual Environments, vol. 13, pp. 395-415, 2004.

[2] S. J. Lederman, R. L. Klatzky, C. L. Hamilton, and G. I. Ramsay, "Perceiving roughness via a rigid probe: Psychophysical effects of exploration speed and mode of touch," Haptics-e: The Electronic Journal for Haptics Research, vol. 1, http://www.haptics-e.org, 1999.

[3] S. A. Wall and W. S. Harwin, "Modelling of surface identifying characteristics using Fourier series," in Proceedings of the ASME Dynamic Systems and Control Division, vol. 67, N. Olgac, Ed. Nashville, TN: ASME, 1999, pp. 65-71.

[4] J. M. Weisenberger, M. J. Krier, and M. A. Rinker, "Judging the orientation of sinusoidal and square-wave virtual gratings presented via 2-DOF and 3-DOF haptic interfaces," Haptics-e: The Electronic Journal for Haptics Research, vol. 1, http://www.haptics-e.org, 2000.

[5] F. W. Campbell and J. G. Robson, "Application of Fourier analysis to the visibility of gratings," Journal of Physiology, vol. 197, pp. 551-566, 1968.

[6] R. Traylor, D. Wilhelm, B. D. Adelstein, and H. Z. Tan, "Design considerations for stand-alone haptic interfaces communicating via UDP protocol," in Proceedings of the 2005 World Haptics Conference (WHC05): The First Joint EuroHaptics Conference and the Symposium on Haptic Interfaces for Virtual Environment and Teleoperator Systems. Pisa, Italy, Mar. 18-20, 2005, pp. 563-564.

[7] H. Z. Tan, B. D. Adelstein, R. Traylor, M. Kocsis, and E. D. Hirleman, "Discrimination of real and virtual highdefinition textured surfaces," in Proceedings of the 14th International Symposium on Haptic Interfaces for Virtual Environment and Teleoperator Systems (HAPTICS '06): IEEE Computer Society, 2006, pp. 3-9.

[8] H. Levitt, "Transformed up-down methods in psychoacoustics," Journal of the Acoustical Society of America, vol. 49, pp. 467-477, 1971.
[9] M. R. Leek, "Adaptive procedures in psychophysical research," Perception \& Psychophysics, vol. 63, pp. 1279-1292, 2001.

[10] S. J. Bolanowski Jr., G. A. Gescheider, R. T. Verrillo, and C. M. Checkosky, "Four channels mediate the mechanical aspects of touch," Journal of the Acoustical Society of America, vol. 84, pp. 1680-1694, 1988.

[11] C. L. Van Doren, "The effects of a surround on vibrotactile thresholds: Evidence for spatial and temporal independence in the non-Pacinian I (NP I) channel," Journal of the Acoustical Society of America, vol. 87, pp. 2655-2661, 1990.

[12] K. O. Johnson, T. Yoshioka, and F. Vega-Bermudez, "Tactile functions of mechanoreceptive afferents innervating the hand," Journal of Clinical Neurophysiology, vol. 17, pp. 539-558, 2000.

[13] A. W. Goodwin and J. W. Morley, "Sinusoidal movement of a grating across the monkey's fingerpad: Representation of grating and movement features in afferent fiber responses," The Journal of Neuroscience, vol. 1987, pp. 2168-2180, 1987.

[14] J. W. Morley, A. W. Goodwin, and I. Darian-Smith, "Tactile discrimination of gratings," Experimental Brain Research, vol. 49, pp. 291-299, 1983.

[15] C. L. Van Doren, "A model of spatiotemporal tactile sensitivity linking psychophysics to tissue mechanics," The Journal of the Acoustical Society of America, vol. 85, pp. 2065-2080, 1989. 